\title{
MAGNETIC MONOPOLES IN UNIFIED GAUGE THEORIES
}

\author{
G. 't HOOFT \\ CERN, Geneva
}

Received 31 May 1974

\begin{abstract}
It is shown that in all those gauge theories in which the electromagnetic group U(1) is taken to be a subgroup of a larger group with a compact covering group, like SU(2) or SU(3), genuine magnetic monopoles can be created as regular solutions of the field equations. Their mass is calculable and of order $137 M_{\mathrm{W}}$, where $M_{\mathrm{W}}$ is a typical vector boson mass.
\end{abstract}

\section{Introduction}

The present investigation is inspired by the work of Nielsen et al. [1], who found that quantized magnetic flux lines, in a superconductor, behave very much like the Nambu string [2]. Their solution consists of a kernel in the form of a thin tube which contains most of the flux lines and the energy; all physical fields decrease exponentially outside this kernel. Outside the kernel we do have a transverse vector potential $A$, but there it is rotation-free: if we put the kernel along the $z$ axis, then

$$
A(\boldsymbol{x}) \propto(y,-x, 0) /\left(x^{2}+y^{2}\right) .
$$

$A(x)$ can be obtained by means of a gauge transformation $\Omega(\varphi)$ from the vacuum. Here $\varphi$ is the angle about the $z$ axis:

$$
\Omega(0)=\Omega(2 \pi)=1 \text {. }
$$

It is obvious that such a string cannot break since we cannot have an end point: it is impossible to replace a rotation over $2 \pi$ continuously by $\Omega(\varphi) \rightarrow 1$. Or: magnetic monopoles do not occur in the system. Also it is easy to see that these strings are oriented: two strings with opposite direction can annihilate; if they have the same direction they may only join to form an even tighter string.

Now, let us suppose that the electromagnetism in the superconductor is in fact described by a unified gauge theory, in which the electromagnetic group U(1) is a subgroup of, say, SO(3). In such a non-Abelian theory one can only imagine nonoriented strings, because a rotation over $4 \pi$ can be continuously shifted towatds a fixed $\Omega$. What happened with our original strings? The answer is simple: in an SO(3) gauge theory magnetic monopoles with twice the flux quantum (i.e., the 
Schwinger $[3,4]$ value), occur. Two of the original strings, oriented in the same direction, can now annihilate by formation of a monopole pair [5].

From now on we shall dispose of the original superconductor with its quantized flux lines. We consider free monopoles in the physical vacuum. That these monopoles are possible, as regular solutions of the field equations, can be understood in the following way. Imagine a sphere, with a magnetic flux $\Phi$ entering at one spot (see fig. 1). Immediately around that spot, on the contour $\mathrm{C}_{0}$ in fig. 1 , we must have a magnetic potential field $\boldsymbol{A}$, with $\oint(\boldsymbol{A} \cdot \mathrm{d} \boldsymbol{x})=\Phi$. It can be obtained from the vacuum by applying a gauge transformation $\Lambda$ :

$$
\boldsymbol{A}=\nabla \Lambda \text {. }
$$

This $\Lambda$ is multivalued. Now we require that all fields, which transform according to

$$
\psi \rightarrow \psi \mathrm{e}^{n i \Lambda},
$$

to remain single valued, so $\Phi$ must be an integer times $2 \pi$ : we then have a complete gauge rotation along the contour in fig. 1 .

In an Abelian gauge theory we must necessarily have some other spot on the sphere where the flux lines come out, because the rotation over $2 k \pi$ cannot continuously change into a constant while we lower the contour $\mathrm{C}$ over the sphere. In a non-Abelian theory with compact covering group, however, for instance the group $O(3)$, a rotation over $4 \pi$ may be shifted towards a constant, without singularity: we may have a vacuum all around the sphere. In other theories, even rotations over $2 \pi$

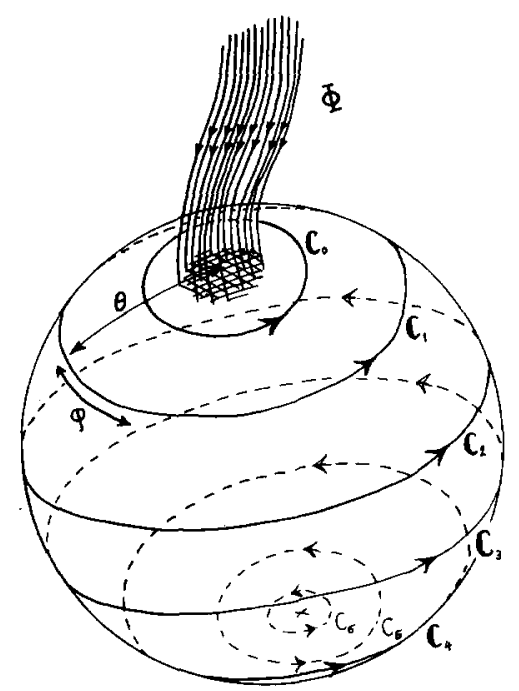

Fig. 1. The contour $C$ on the sphere around the monopole. We deplace it from $C_{0}$ to $C_{1}$, etc., until it shrinks at the bottom of the sphere. We require that there be no singularity at that point. 
may be shifted towards a constant. This is why a magnetic monopole with twice or sometimes once the flux quantum is allowed in a non-Abelian theory, if the electromagnetic group $\mathrm{U}(1)$ is a subgroup of a gauge group with compact covering group. There is no singularity anywhere in the sphere, nor is there the need for a Dirac string.

This is how we were led to consider solutions of the following type to the classical field equations in a non-Abelian Higgs-Kibble system: a small kernel occurs in the origin of three dimensional space. Outside that kernel a non-vanishing vector potential exists (and other non-physical fields) which can be obtained from the vacuum* by means of a gauge transformation $\Omega(\theta, \varphi)$. At one side of the sphere $(\cos \theta \rightarrow 1)$ we have a rotation over $4 \pi$, which goes to unity at the other side of the sphere $(\cos \theta \rightarrow-1)$. For such a rotation one can, for instance, take the following $\mathrm{SU}(2)$ matrix:

$$
\Omega(\theta, \varphi)=\cos \frac{1}{2} \theta\left(\begin{array}{ll}
\mathrm{e}^{i \varphi} & 0 \\
0 & \mathrm{e}^{-i \varphi}
\end{array}\right)+\sin \frac{1}{2} \theta\left(\begin{array}{ll}
0 & i \\
i & 0
\end{array}\right) .
$$

Now consider one rotation of the angle $\varphi$ over $2 \pi$. At $\theta=0$, this $\Omega$ rotates over $4 \pi$ (the spinor rotates over $2 \pi$ ). At $\theta=\pi$, this $\Omega$ is a constant. One easily checks that

$$
\Omega \Omega^{\dagger}=1 \text {. }
$$

In the usual gauge theories one normally chooses the gauge in which the Higgs field is a vector in a fixed direction, say, along the positive $z$ axis, in isospin space. Now, however, we take as a gauge condition that the Higgs field is $\Omega(\theta, \varphi)$ times this vector. As we shall see in sect. 2, this leads to a new boundary condition at infinity, to which corresponds a non-trivial solution of the field equations: a stable particle is sitting at the origin. It will be shown to be a magnetic monopole. If we want to be conservative and only permit the normal boundary condition at infinity, with Higgs fields pointing in the $z$ direction, then still monopole-antimonopole pairs, arbitrarily far apart, are legitimate solutions of the field quations.

\section{The model}

We must have a model with a compact covering group. That, unfortunately, excludes the popular SU(2) $\times$ U(1) model of Weinberg and Salam [6]. There are two classes of possibilities.

(i) In models of the type described by Georgi and Glashow [7], based on SO(3), we can construct monopoles with a mass of the order of $137 M_{\mathrm{W}}$, where $M_{\mathrm{W}}$ is the

\footnotetext{
* As we shall see this vacuum will still contain a radial magnetic field. This is because the incoming field in fig. 1 will be spread over the whole sphere.
} 
mass of the familiar intermediate vector boson. In the Georgi-Glashow model, $M_{\mathrm{W}}<53 \mathrm{GeV} / c^{2}$.

(ii) The Weinberg-Salam model can still be a good phenomenological description of processes with energies around hundreds of $\mathrm{GeV}$, but may need extension to a larger gauge group at still higher energies. Weinberg [8] proposed SU(3) $\times S U(3)$ which would then be compact. Then the monopole mass would be 137 times the mass of one of the superheavy vector bosons.

We choose the first possibility for our sample calculations, because it is the simplest one. We take as our Lagrangian:

$$
\mathcal{L}=-\frac{1}{4} G_{\mu \nu}^{a} G_{\mu \nu}^{a}-\frac{1}{2} D_{\mu} Q_{a} D_{\mu} Q_{a}-\frac{1}{2} \mu^{2} Q_{a}^{2}-\frac{1}{8} \lambda\left(Q_{a}^{2}\right)^{2},
$$

where

$$
\begin{aligned}
& G_{\mu \nu}^{a}=\partial_{\mu} W_{\nu}^{a}-\partial_{\nu} W_{\mu}^{a}+\mathrm{e} \epsilon_{a b c} W_{\mu}^{b} W_{\nu}^{c}, \\
& D_{\mu} Q_{a}=\partial_{\mu} Q_{a}+\mathrm{e} \epsilon_{a b c} W_{\mu}^{b} Q_{c} .
\end{aligned}
$$

$W_{\mu}^{a}$ and $Q_{a}$ are a triplet of vector fields and scalar fields, respectively.

We choose the parameter $\mu^{2}$ to be negative so that the field $Q$ gets a non-zero vacuum expectation value $[6,7,9]$ :

$$
\left\langle Q_{a}\right\rangle^{2}=F^{2}, \quad \mu^{2}=-\frac{1}{2} \lambda F^{2} .
$$

Two components of the vector field will acquire a mass:

$$
M_{\mathrm{W}_{1,2}}=e F \text {, }
$$

whereas the third component describes the surviving Abelian electromagnetic interactions. The Higgs particle has a mass:

$$
M_{\mathrm{H}}=\sqrt{\lambda} F \text {. }
$$

We are interested in a solution where the Higgs field is not rotated everywhere towards the positive $z$ direction. If we apply the transformation $\Omega$ of eq. (1.5) to the isospin-one vector $F(0,0,1)$ we get

$$
F(\sin \theta \cos \varphi, \sin \theta \sin \varphi, \cos \theta) .
$$

We shall take this isovector as our boundary condition for the Higgs field at spacelike infinity. As one can easily verify, it implies that the Higgs field must have at least one zero. This zero we take as the origin of our coordinate system.

We now ask for a solution of the field equations that is time-independent and spherically symmetric, apart from the obvious angle dependence. Introducing the vector

$$
r_{a}=(x, y, z), \quad r_{a}^{2}=r^{2},
$$


we can write

$$
Q_{a}(x, t)=r_{a} Q(r), \quad W_{\mu}^{a}(x, t)=\epsilon_{\mu a b} r_{b} W(r),
$$

where $\epsilon_{\mu a b}$ is the usual $\epsilon$ symbol if $\mu=1,2,3$, and $\epsilon_{4 a b}=0$.

In terms of these variables the Lagrangian becomes

$$
\begin{aligned}
L= & \int \mathcal{L} \mathrm{d}^{3} x=4 \pi \int_{0}^{\infty} r^{2} \mathrm{~d} r\left[-r^{2}\left(\frac{\mathrm{d} W}{\mathrm{~d} r}\right)^{2}-4 r W \frac{\mathrm{d} W}{\mathrm{~d} r}-6 W^{2}-2 e r^{2} W^{3}\right. \\
& -\frac{1}{2} e^{2} r^{4} W^{4}-\frac{1}{2} r^{2}\left(\frac{\mathrm{d} Q}{\mathrm{~d} r}\right)^{2}-r Q \frac{\mathrm{d} Q}{\mathrm{~d} r}-\frac{3}{2} Q^{2}-2 e r^{2} W Q^{2}-e^{2} r^{4} W^{2} Q^{2} \\
& \left.+\frac{1}{4} \lambda F^{2} r^{2} Q^{2}-\frac{1}{8} \lambda r^{4} Q^{4}-\frac{1}{8} \lambda F^{4}\right],
\end{aligned}
$$

where the constant has been added to give the vacuum a vanishing action integral. The field equations are obtained by requiring $L$ to be stationary under small variations of the functions $W(r)$ and $Q(r)$. The energy of the system is then given by

$$
E=-L \text {, }
$$

since our system is stationary.

Before calculating this energy, let us concentrate on the boundary condition at $r \rightarrow \infty$. From the preceding arguments we already know that we must insist on

$$
Q(r) \rightarrow F / r \text {. }
$$

The field $W$ must behave smoothly, as some negative power of $r$ :

$$
W(r) \rightarrow a r^{-n} \text {. }
$$

From (2.9) we find the Lagrange equation

$$
\begin{aligned}
& \frac{\mathrm{d}}{\mathrm{d} r}\left(2 r^{4} \frac{\mathrm{d} W}{\mathrm{~d} r}+4 r^{3} W\right) \\
& \quad=r^{2}\left[4 r \frac{\mathrm{d} W}{\mathrm{~d} r}+12 W+6 e r^{2} W^{2}+2 e^{2} r^{4} W^{3}+2 e r^{2} Q^{2}+2 e^{2} r^{4} W Q^{2}\right] .
\end{aligned}
$$

So, substituting (2.11) and (2.12),

$$
\begin{aligned}
& (3-n)(4-2 n) a r^{2-n} \underset{r \rightarrow \infty}{\longrightarrow}-4 n a r^{2-n}+12 a r^{2-n}+6 e a^{2} r^{4-2 n}+2 e^{2} a^{3} r^{6-3 n} \\
& +2 e F^{2} r^{2}+2 e^{2} a F^{2} r^{4-n}
\end{aligned}
$$

The only solution is

$$
n=2, \quad a=-1 / e .
$$

So, far from the origin, the fields are 


$$
W_{\mu}^{a}(x, t) \rightarrow-\epsilon_{\mu a b} r_{b} / e r^{2}, \quad Q_{a}(x, t) \rightarrow F r_{a} / r
$$

Now most of these fields are not physical. To find the physically observable fields, in particular the electromagnetic ones, $F_{\mu \nu}$, we must first give a gauge invariant definition, which will yield the usual definition in the gauge where the Higgs field lies along the $z$ direction everywhere. We propose:

$$
F_{\mu \nu}=\frac{1}{|Q|} Q_{a} G_{\mu \nu}^{a}-\frac{1}{e|Q|^{3}} \epsilon_{a b c} Q_{a}\left(D_{\mu} Q_{b}\right)\left(D_{\nu} Q_{c}\right),
$$

because, if after a gauge rotation, $Q_{a}=|Q|(0,0,1)$ everywhere within some region, then we have there

$$
F_{\mu \nu}=\partial_{\mu} W_{\nu}^{3}-\partial_{\nu} W_{\mu}^{3},
$$

as one can easily check. (Observe that the definition (2.17) satisfies the usual

Maxwell equations, except where $Q^{a}=0$; this is one other way of understanding the possibility of monopoles in this theory.) From (2.16), we get (see the definitions $(2.2))$ :

$$
\begin{aligned}
& Q_{a} G_{\mu \nu}^{a}=-\frac{F}{e r^{3}} \epsilon_{\mu \nu a} r_{a}, \\
& D_{\mu} Q_{a}=\partial_{\mu} Q_{a}+e \epsilon_{a b c} W_{\mu}^{b} Q_{c}=0 .
\end{aligned}
$$

Hence

$$
F_{\mu \nu}=-\frac{1}{e r^{3}} \epsilon_{\mu \nu a} r_{a} .
$$

Again, the $\epsilon$ symbol has been defined to be zero as soon as one of its indices has the value 4 . So, there is a radial magnetic field

$$
B_{a}=r_{a} / e r^{3}
$$

with a total flux

$$
4 \pi / e \text {. }
$$

Hence, our solution is a magnetic monopole, as we expected. It satisfies Schwinger's condition

$$
e g=1
$$

(in units where $\hbar=1$ ). In sect. 4 , however, we show that in certain cases only Dirac's condition

$$
e g=\frac{1}{2} n, \quad n \text { integer },
$$

is satisfied. 


\section{The mass of the monopole}

Let us introduce dimensionless parameters:

$$
\begin{array}{ll}
w=W / F^{2} e, & q=Q / F^{2} e, \\
x=e F r, & \beta=\lambda / e^{2}=M_{\mathrm{H}}^{2} / M_{\mathrm{W}}^{2} .
\end{array}
$$

From (2.9) and (2.10) we find that the energy $E$ of the system is the minima! value of

$$
\begin{aligned}
& \frac{4 \pi M_{\mathrm{W}}}{e^{2}} \int_{0}^{\infty} x^{2} \mathrm{~d} x\left[x^{2}\left(\frac{\mathrm{d} w}{\mathrm{~d} x}\right)^{2}+4 x w \frac{\mathrm{d} w}{\mathrm{~d} x}+6 w^{2}+2 x^{2} w^{3}+\frac{1}{2} x^{4} w^{4}+\frac{1}{2} x^{2}\left(\frac{\mathrm{d} q}{\mathrm{~d} x}\right)^{2}\right. \\
& \left.+x q \frac{\mathrm{d} q}{\mathrm{~d} x}+\frac{3}{2} q^{2}+2 x^{2} w q^{2}+x^{4} w^{2} q^{2}-\frac{1}{4} \beta x^{2} q^{2}+\frac{1}{8} \beta x^{4} q^{4}+\frac{1}{8} \beta\right] .
\end{aligned}
$$

The quantity between the brackets is dimensionless and the extremum can be found by inserting trial functions and adjusting their parameters.

We found that the mass of the monopole (which is equal to the energy $E$ since the monopole is at rest) is

$$
M_{\mathrm{m}}=\frac{4 \pi}{e^{2}} M_{\mathrm{W}} C(\beta),
$$

where $C(\beta)$ is nearly independent of the parameter $\beta$. It varies from 1.1 for $\beta=0.1$ to 1.44 for $\beta=10^{*}$.

Only in the Georgi-Glashow model (for which we did this calculation) is the parameter $M_{\mathrm{W}}$ in eq. (3.3) really the mass of the conventional intermediate vector boson. In other models it will in general be the mass of that boson which corresponds to the gauge transformations of the compact covering group: some of the superheavies in Weinberg's $S U(3) \times S U(3)$ for instance.

\section{Conclusions}

The relation between charge quantization and the possible existence of magnetic monopoles has been speculated on for a long time [10] and it has been observed that the gauge theories with compact gauge groups provide for the necessary charge quantization [11]. On the other hand, solutions of the field equations with abnormally rotated boundary conditions for the Higgs fields have also been considered before $[1,12]$. Nevertheless, it had escaped to our notion until now that magnetic monopoles occur among the solutions in those theories, and that their properties are predictable and calculable.

* These values may be slightly too high, as a consequence of our approximation procedure. 
Our way of formulating the theory of magnetic monopoles avoids the introduction of Dirac's string [3], We expect no fundamental problems in calculating quantum corrections to the solution although they might be complicated to carry out.

The prediction is the most striking for the Georgi-Glashow model, although even in that model the mass is so high that that might explain the negative experimental evidence so far. If Weinberg's $S U(2) \times U(1)$ model wins the race for the presently observed weak interactions, then we shall have to wait for its extension to a compact gauge model, and the predicted monopole mass will be again much higher. Finally, one important observation. In the Georgi-Glashow model, one may introduce isospin $\frac{1}{2}$ representations of the group SU(2) describing particles with charges $\pm \frac{1}{2} e$. In that case our monopoles do not obey Schwinger's condition, but only Dirac's condition

$$
q g=\frac{1}{2}
$$

where $q$ is the charge quantum and $g$ the magnetic pole quantum, in spite of the fact that we have a completely quantized theory. Evidently, Schwinger's arguments do not hold for this theory [13]. We do have, in our model

$$
\Delta q g=1,
$$

where $\Delta q$ is the charge-difference between members of a multiplet, but this is certainly not a general phenomenon. In Weinberg's SU(3) $\times$ SU(3) the monopole quantum is the Dirac one and in models where the leptons form an SU(3) $\times$ SU(3) octet [14] the monopole quantum is three times the Dirac value (note the possibility of fractionally charged quarks in that case).

We thank H. Strubbe for help with a computer calculation of the coefficient $C(\beta)$, and B. Zumino and D. Gross for interesting discussions.

\section{References}

[1] H.B. Nielsen and P. Olesen, Niels Bohr Institute preprint, Copenhagen (May 1973); B. Zumino, Lectures given at the 1973 Nato Summer Institute in Capri, CERN preprint TH. 1779 (1973).

[2] Y. Nambu, Proc. Int. Conf. on symmetries and quark models, Detroit, 1969 (Gordon and Breach, New York, 1970) p. 269;

L. Susskind, Nuovo Cimento 69A (1970) 457;

J.L. Gervais and B. Sakita, Phys. Rev. Letters 30 (1973) 716.

[3] P.A.M. Dirac, Proc. Koy. Soc. A133 (1934) 60; Phys. Rev. 74 (1948) 817.

[4] J. Schwinger, Phys. Rev. 144 (1966) 1087.

[5] G. Parisi, Columbia University preprint CO-2271-29.

[6] S. Weinberg, Phys. Rev. Letters 19 (1967) 1264.

[7] M. Georgi and S.L. Glashow, Phys. Rev. Letters 28 (1972) 1494.

[8] S. Weinberg, Phys. Rev. D5 (1972) 1962. 
[9] F. Englert and R. Brout, Phys. Rev. Letters 13 (1964) 321;

P.W. Higgs, Phys. Letters 12 (1964) 132; Phys. Rev. Letters 13 (1964) 508; Phys. Rev. 145 (1966) 1156 ;

G.S. Guralnick, C.R. Hagen and T.W.B. Kibble, Phys. Rev. Letters 13 (1964) 585.

[10] D.M. Stevens, Magnetic monopoles: an updated bibliography, Virginia Poly. Inst. and State University preprint VPI-EPP-73-5 (October 1973).

[11] C.N. Yang, Phys, Rev. D1 (1970) 2360.

[12] A. Neveu and R. Dashen, Private communication.

[13] B. Zumino, Strong and weak interactions, 1966 Int. School of Physics, Erice, ed.

A. Zichichi (Acad. Press, New York and London) p. 711.

[14] A. Salam and J.C. Pati, University of Maryland preprint (November 1972). 